\title{
CAMINOS CRUZADOS: GÉNERO EN LAS POLÍTICAS EDUCATIVAS EN EL PERÚ EN LOS ÚLTIMOS DIEZ AÑOS
}

\author{
FAnNi Muñoz Cabrejo*
}

\begin{abstract}
Lo femenino no está llamado a construir un mundo similar al del hombre: mundo violento, inquietante, que existe a través de una dominación impuesta a la naturaleza, a los animales, a los otros humanos. Devenir ella misma un mundo, cultivarse sin violencia ni poder sobre lo que la rodea, corresponde más al ser de la mujer (...). El devenir humano se cumple de manera diversa del hombre occidental, que "sale con violencia de su morada y que ara, captura, doma”. La cultura corresponde entonces a un dominio de sí más que a una salida de sí para dominar a los otros e imponerles un orden tal vez menos armonioso que el orden natural inicial.
\end{abstract}

Luce Irigarayay (1998, p. 88)

RESUMEN: El estudio examina las políticas educativas producidas en el Perú entre los años de 1996 a 2003, desde una perspectiva de género. A través de una revisión y análisis de la normatividad y documentación producida en el periodo, muestra cómo las políticas educativas inscritas en el paradigma de la igualdad no incorporaron una perspectiva de género de manera explícita. Existieron acciones individuales y puntuales, no necesariamente integradas entre sí, como los lineamientos de política educativa curricular, materiales educativos del nivele primaria y acciones en capacitación docente. Sin embargo, cuando se hace referencia a la noción de género en la educación se asume que no existen mayores brechas de género por-

* Doctorado en Historia por el Centro de Estudios Históricos de El Colegio de México y docente de la Facultad de Ciencias Sociales de la Pontificia Universidad Católica del Perú. E-mail: famunoz@pucp.edu.pe

Disponível em <http://www.cedes.unicamp.br> 
Caminos cruzados: Género en las políticas educativas en el Perú en los últimos diez años

que la cobertura en la matricula escolar es alta tanto en las mujeres como en los hombres. Finalmente, durante los primeros años del XXI, en un nuevo paradigma de las políticas de equidad, la perspectiva de género es retomada pero nuevamente bajo el rótulo de las poblaciones vulnerables.

Palabras clave: Educación. Políticas Públicas. Igualdad. Currículum.

CROSSING WAYS: GENDER IN THE EDUCATIONAL POLICIES

of Peru these ten last years

ABSTRACT: This paper explores the educational policies adopted in Peru in the 1990s and the first years of the XXI ${ }^{\text {st }}$ century (19962003), from a gender point of view. Reviewing and analyzing the standards and documentation from that period, it shows how educational policies claiming an equality paradigm did not explicitly incorporate gender issues. Some individual and singular actions that were not necessarily linked took place, such as educational curriculum policy guidelines, some primary level educational materials and some initiatives in teacher training. However, whenever the gender concept in education is referred to, it is assumed that there are no major gender breeches since the male school registration is as high as the female one. Finally, the gender perspective only reappeared, although, once again, under the label of vulnerable population, during the early $\mathrm{XXI}^{\mathrm{st}}$ century, and within a new paradigm of policies of equality.

Key words: Gender. Education. Public policies. Equality. Curriculum.

$\mathcal{L}$

a presente investigación ${ }^{1}$ examina las políticas educativas producidas en el Perú durante la década de los noventa y los primeros años del siglo XXI (1996-2003), desde una perspectiva de género. ¿De qué se está hablando cuando se emplea la noción de equidad de género en la educación? ¿Qué hacer para favorecerla? Preguntarse por la equidad es empezar a construir un horizonte de acción/interpretación que debe tomar en cuenta distintas perspectivas y enfoques de trabajo. Es necesario pensarla, entonces, desde múltiples miradas y voces.

Sobre la base de una propuesta metodológica común que comprende entrevistas a funcionarios y personas involucradas en la formulación de las políticas educativas, así como el análisis de la normatividad, la revisión de programas, contenidos curriculares y materiales 
educativos, se trata de analizar lo que se avanzó en el caso peruano, en cuanto a la inclusión de una perspectiva de género, durante este periodo de reformas educativas.

Igualdad y equidad en las políticas peruanas: una mirada a la normatividad

En la década de 1990, el tema educativo adquiere relevancia sin llegar a ubicarse dentro de un encuadre institucional y doctrinario que tuvo durante la década de 1970. Esto, bajo un modelo de Estado neoliberal, populista y dictatorial promovido durante el gobierno de Alberto Fujimori.

Las acciones de reforma educativa que comenzaron a implementarse a mediados de 1995, comprendieron: la renovación curricular, una nueva propuesta de estructura del sistema educativo, la modernización de la gestión, la implementación de un sistema de formación y capacitación docente, entre otras. ${ }^{2}$ Si bien la reforma curricular comprendió los distintos niveles y modalidades educativas, su implementación se centró en el nivel de primaria. Cabe anotar que estas acciones de reforma, más que responder a las demandas de los y las ciudadanas, fueron impulsadas por las agencias multilaterales y las cooperaciones internacionales en el marco de los procesos de ajuste bajo una economía neoliberal.

Las acciones de reforma educativa se realizaron en un contexto de reinserción del Perú en la economía mundial. Es en este sentido que se explica la centralidad que tuvo para el gobierno de Fujimori suscribirse a los acuerdos de las conferencias y cumbres mundiales de las $\mathrm{Na}$ ciones Unidas. ${ }^{3}$ En cierto sentido, este gobierno trató de cumplir con los acuerdos consagrados en las distintas conferencias y convenciones tales como la Conferencia de Jomtien (Tailandia, 1990) en la que se aprueba la Declaración Mundial sobre Educación para Todos. En esta conferencia el Perú se comprometió a cumplir con el derecho universal a la educación.

En muchos de estos eventos se promovió la defensa de los derechos de las mujeres, su desarrollo personal y su participación en la esfera de la vida pública y en la toma de decisiones. Este es el caso de la Cumbre Internacional sobre Población y Desarrollo (El Cairo, 1994), 
la Conferencia de Derechos Humanos de las Mujeres (Viena, 1994), la IV Conferencia Internacional sobre la Mujer (Beijing, 1995), el Foro Mundial de Educación (Dakar, 2000), la Cumbre del Milenio de las Naciones Unidas (Nueva York0, 2000) y la Conferencia Regional sobre Educación para Todos (Santo Domingo, 2002).

Pero, ¿cuál fue el campo de actuación para tratar el tema de género? ¿Con qué soportes normativos se contó? Un primer aspecto que hay que señalar es que reforma curricular y todas las acciones de cambios que implicaron los intentos de modernización de la educación se realizaron dentro del marco normativo de la LGE-1982, la cual correspondía a otro periodo y contexto sociopolítico. Es por ello que como antecedente nos remitimos a la misma.

La LGE-1982 señala que la educación se sujeta a las normas básicas relacionadas con la obligatoriedad de la educación primaria, la gratuidad de la educación y la atención preferente a sectores marginados, zonas de frontera y población en área rural. Asimismo, se rechaza toda forma de exclusión (Diario Oficial el Peruano, 1982, artículo 4º).

Así, esta ley estaba orientada a garantizar el acceso de la población a la educación, especialmente a la educación primaria; es decir, a garantizar la universalización de la educación. Es en tanto se cumpla este objetivo que se atiende a las mujeres. La Ley enfatiza en el derecho de todo ser humano a ser educado y a las condiciones materiales que permitan un proceso educativo satisfactorio.

Si bien la Ley de Educación del 82 fue el marco bajo el cual se normó la educación, durante la década del noventa, en la medida que el Perú - como se ha señalado - comienza a retomar su presencia en los foros y conferencias internacionales, la noción de género se introduce en el ámbito educativo a partir de 1990 con Jomtien, aspecto que se reforzará con los acuerdos de Beijing en 1995.

Pese a que el gobierno peruano se comprometió a cumplir los acuerdos de Educación para Todos de Jomtien, las acciones y mecanismos para que se cumplan, no solo no se crearon sino que las pocas medidas que se crearon fueron lentas. Así el establecimiento de una comisión para trabajar sobre Educación para Todos recién se formó en 1999, con la preparación de un diagnóstico sobre la situación de la educación peruana para la conferencia de Dakar, donde se evaluarían los avances de todos los países que asumieron un compromiso en Jomtiem. ${ }^{4}$ 
En la documentación producida por el sector educación entre 1995 y el 2001 se puede observar que estos acuerdos se convirtieron en algunas acciones poco articuladas para atender los problemas vinculados a la mujer; evidenciándose que el enfoque de género no fue un eje de acción prioritario en este período. Curiosamente, cuando se hace referencia a esa noción es para afirmar que no existen problemas abordables desde esta perspectiva en la educación peruana dadas las altas tasas de cobertura en la matrícula escolar en primaria, tanto de hombres como de mujeres.

Así, para el sector educación en el Perú el género aparece como una brecha superada, un problema resuelto. Esto se explicita en algunos documentos oficiales producidos por el sector para referirse a las cifras sobre cobertura. Así mismo, muchos de los funcionarios del sector y de los ex funcionarios que trabajaron durante la década de 1990 son enfáticos en señalar que en el sistema educativo peruano no existen problemas de género. La poca reflexión sobre este concepto y sus potencialidades tiene como resultado su simplificación y un uso que, en cierto sentido, sirve para reafirmar las exclusiones y jerarquías de la sociedad peruana. El género es entendido exclusivamente como el comportamiento de los sexos, homologándolo a una característica biológica.

El diagnóstico del sector educativo realizado para Dakar fue negativo con relación a la inclusión del tema de género, pero cabe destacar que durante los primeros años de la década de 1990 se llevaron a cabo acciones puntuales que incluyeron este tema. Esto guarda correspondencia con el hecho de que desde el gobierno se enunciaba la importancia de la participación de las mujeres en la escena pública, que, como hemos visto anteriormente, se tradujo en programas concretos, nuevas instituciones y medidas dirigidas a las mujeres (Blondet, 2002).

Es en este marco que la noción de género comienza a ser introducida en los lineamientos de política educativa, específicamente en los lineamientos curriculares, cuando se especifica que uno de los cinco ejes transversales del nuevo enfoque curricular por competencias es el denominado Población, familia y sexualidad; categorías que eran relacionadas con el género. Curiosamente, la transversalidad de género fue una de las estrategias acordadas en Beijing (Stromquist, 2003, p. 4). La participación de Fujimori en la IV Conferencia Internacional de la Mujer marcó un hito con relación a la atención que le dedicó el gobierno a la participación de las mujeres en la vida pública. Como observó Maruja Barrig, 
durante esta década la visibilidad de las mujeres emergía con fuerza como producto de los "procesos de modernización de los estados y la presión de los movimientos de mujeres" (2000, p. 1). Esto se materializa en la creación de distintas instancias estatales para apoyar a las mujeres en el reconocimiento de sus derechos. Así, por ejemplo, en el Congreso se conforma la Comisión de la Mujer (1996) y en la Defensoría del Pueblo se crea la Defensoría Especializada en Derechos de la Mujer (1996). En ambos casos, la presión de los grupos feministas fue fundamental. Otro hecho clave fue la constitución del Ministerio de Promoción de la Mujer y del Desarrollo Humano (PROMUDeh), hoy Ministerio de la Mujer y del Desarrollo Social (MIMDES). Este Ministerio se encarga de normar y ejecutar las políticas y programas de promoción de la mujer.

Asimismo, un intento por cumplir con los convenios internacionales, en el marco de la política nacional de población, fue la creación del Programa de Educación Sexual en el Ministerio de Educación en 1996, en el que se elaboraron las Guías de Educación Sexual. El diseño de las guías contó con la participación de grupos feministas pero, luego de su distribución, estas fueron confiscadas y retiradas de circulación por presión de la Iglesia Católica, institución que luego fue encargada de la elaboración de nuevas guías. Inicialmente, el programa de capacitación a docentes con las guías se dirigió al nivel de secundaria, pero después se trabajó con el nivel de primaria y con los Institutos Superiores Pedagógicos. El programa fue concebido para que los docentes lo incorporasen dentro del currículo tanto de primaria como el de secundaria.

\section{Educación y desarrollo democrático: irrupción de la equidad}

A partir de la primera década del 2000 se presenta un cambio acelerado en la política educativa, todo en ello en un contexto de unta transición democrática. Las propuestas educativas comienzan a ser abiertas a la discusión y debate nacional. La definición de las políticas de Estado a través del Acuerdo Nacional (2001) y la elaboración de la nueva Ley de Educación constituyen hitos fundamentales (2003). No obstante los cambios presentes en estos marcos, también se observan líneas de continuidad con las acciones de reforma iniciadas en la década de los 90s. 
Este discurso de la equidad, como señala Néstor López, se introduce a finales de la década de 1990, en el discurso oficial de las políticas educativas. Como noción "irrumpe en un campo donde la concepción igualitarista de la educación está instalada en la base de los sistemas de la región desde sus orígenes" (López 2005, p. 64). La equidad se vincula a la pobreza y vulnerabilidad que ocupan un lugar central en la definición de las políticas sociales.

La política del Acuerdo Nacional dedicada a la educación, ${ }^{5}$ como la Ley General de la Educación (LGE-2003), señalan que el Estado asegurará una educación gratuita, universal y de calidad que promueva la equidad entre hombres y mujeres. La equidad entre hombres y mujeres es concebida una vez más como acceso, enfatizándose el igualitarismo en la calidad educativa. Pero en estos marcos normativos también la falta de equidad entre hombres y mujeres es concebida como "inequidad de género" (Acuerdo Nacional, 2002: Décima Política de Estado). Bajo este enunciado se equipara género con sexo y el problema es el acceso. El género se define en relación al derecho de igualdad de oportunidades entre los sexos. La equidad entre hombres y mujeres es concebida una vez más como acceso, enfatizándose el igualitarismo en la calidad educativa.

También, el año 2002, se instala el Foro Nacional de Educación para Todos, que contó con la participación de representantes de la sociedad civil con la finalidad de asegurar el cumplimiento de Dakar. Este Plan, concebido como una de las dimensiones del Plan Estratégico del Sector Educación en un marco de política a largo plazo, ha sido formulado tres veces como documento base, el último de los cuales se encuentra en una fase de consulta nacional. Este hecho evidencia el escaso interés del Estado por sujetarse a los compromisos internacionales. En el documento base de circulación restringida, publicado por el $\mathrm{Mi}$ nisterio de Educación el año 2003, se explícita que el tema de la equidad será transversal a la educación primaria. ${ }^{6}$ Asimismo, se señala que las políticas educativas con relación a la equidad deben enfatizar en la disminución de las brechas educativas entre la población rural y urbana (Ministerio de Educación, 2003a, p. 49). Entre los fundamentos y los principios que indica el segundo documento interno producido por el Foro, se señala el de la Equidad. Esta es entendida como "atención a la diversidad, garantizar a las personas igualdad de oportunidades de acceso y permanencia en el sistema educativo y contribuye a reducir la exclusión, las inequidades y la pobreza" (Ministerio de Educación, 
2003 , p. 21). Por otro lado, se señala el hecho el hecho de que no existen diferencias sustanciales de género en cuanto al acceso al sistema. Se señala que las diferencias son las que tienen que ver con el nivel de escolarización y el porcentaje de hombres y mujeres que concluyen la educación primaria en los tiempos establecidos (Ministerio de Educación, 2003a). La noción de género que se presenta se limita así al acceso y a la población vulnerable, entre las cuales se enfoca a las mujeres que tienen bajos niveles de escolarización (Ministerio de Educación, 2003a). En el documento se equipara sexo y género, es decir hay un uso biologicista de este concepto.

\section{Ley General de Educación del 2003 (LGE-2003)}

La Ley General de Educación se hizo casi ocho años después de iniciadas las acciones de reforma en el sector. En la formulación de esta Ley participaron distintos actores de la sociedad civil por medio de consultas nacionales. La ley está regida por tres principios: universalidad, equidad y calidad, y propone la participación activa de la sociedad en el sistema educativo y en la formulación de políticas educativas. Esta Ley resulta muy favorable para tratar el tema de género a partir del principio de equidad, no obstante, esta potencialidad no se desarrolla.

Este nuevo dispositivo legal, que surge como resultado de un proceso en el que la participación ciudadana fue fundamental (Congreso de la República del Perú, 2002), debe definir los lineamientos de política educativa del sector y las acciones a implementar. La ley está centrada en un nuevo modelo de relación entre Estado y sociedad en el que ambos asumen responsabilidades específicas frente a la educación (Diario Oficial El Peruano, 2003d, art. $3^{\circ}$ ). También plantea en forma muy clara su concepción de género.

En la LGE-2003, el tema de género se identifica explícitamente con el tema de la equidad, que está incluido como uno de los principios que guiarán a la educación peruana, junto con los de universalización y calidad. En cuanto al principio de equidad en la educación se señala que: Se trata de impulsar proyectos educativos "tendientes a revertir situaciones de desigualdad y/o inequidad por motivo de género, etnias, idioma, condición económica, religión, edad o de cualquier otra índole" (Diario Oficial El Peruano, 2003, art. 18º). Así, la equidad está 
relacionada con la desigualdad de oportunidades en cuanto al ejercicio del derecho a la educación. Se hace una mención explícita a las políticas compensatorias como una de las estrategias mediante las cuales este principio se cumpliría. Así mismo, el principio de equidad se relaciona directamente con poblaciones vulnerables. Entre las medidas de equidad - en las que se incluye el tema de género - se identifica políticas de acción positiva, programas que reviertan situaciones de desigualdad de género y políticas de compensación, entre otras.

La perspectiva de género utilizada está referida casi exclusivamente al acceso al sistema educativo y a la garantía de un desempeño exitoso en éste. El concepto género no hace referencia a la posibilidad de que el sistema educativo reproduzca dentro de sí mismo el sistema de relaciones de poder que sustenta las jerarquías y desigualdades. Creemos que el acceso y permanencia exitosa en el sistema educativo no es una garantía del cuestionamiento de patrones de exclusión que se expresan en y sustentan las relaciones jerárquicas y excluyentes entre los géneros, ya que éste los podría reproducir dentro de sí. Solo si a la equidad se le añade el cuestionamiento de dicho sistema, su apertura al cambio a partir de la inclusión de grupos e individuos diferentes, con distintas expectativas, necesidades etc. El concepto género podría ser innovador frente al ya criticado sexismo.

Aunque existe una gran potencialidad en la tendencia de esta ley para la inclusión de un enfoque de género no escencialista, que reivindique la diversidad; el tema se presenta limitado y centrado específicamente en las poblaciones vulnerables. Bajo el paradigma de la equidad, se reconoce que el acceso universal al sistema educativo no será suficiente para lograr la igualdad entre los sexos. Los estereotipos de género, el trato diferenciado, la subrepresentación de las mujeres serán entendidos como limitantes de esta meta. La noción de equidad empezará a ser utilizada, entonces, para afrontar una desventaja histórica y a la vez focalizada en el horizonte de la igualdad. Se empezará a hacer referencia a la igualdad de oportunidades y a las politicas compensatorias para las mujeres, enfatizando en aquellas históricamente más vulnerables: las mujeres rurales.

Restringir la equidad de género en la educación al horizonte de la igualdad limita el potencial crítico y propositivo del enfoque de género para la búsqueda de una sociedad más justa. El sistema educativo peruano está constituido sobre una matriz jerárquica, androcéntrica y exclu- 
yente de la diversidad que es preciso afrontar desde las políticas educativas. En este sentido, creemos que es necesario incluir en el concepto de equidad lo que Nancy Fraser (1997) denomina dimensión de reconocimiento de la justicia social. En esta dimensión, "la meta, en su forma más plausible es un mundo abierto a la diferencia, en el cual la asimilación a una mayoría o a normas culturalmente predominantes no es el precio que debe pagarse para obtener igual respeto" (Fraser, 1997, p. 9). Pensar este concepto en el horizonte del reconocimiento positivo de la diversidad propuesto por la CVR implicará no solo atender a la diversidad como camino hacia la igualdad, sino como valiosa en sí misma. Se trata de reconocer los aportes de los otros - desde su particularidad étnicocultural y de género - al sistema educativo y a la sociedad peruana.

Un aspecto que cabe señalar es que los convenios y conferencias internacionales desarrollados en la última década se inscriben mayormente al interior del denominado "feminismo de la igualdad". Buscan realzar el lugar de la mujer y luchar porque estas obtengan iguales derechos que los hombres. Es por ello que se insiste en la igualdad de oportunidades de hombres y mujeres en la educación básica y el efecto positivo que tiene educar a las mujeres para reducir la pobreza. Pese a que desde la conferencia de El Cairo se comienza a hablar de la equidad, esta mayormente está asociada a la igualdad. Este es también un elemento central de los objetivos de Educación para Todos (Dakar, 2000), pero en este caso se enfatiza en población menos favorecida, es decir mujeres rurales y pobres. Existe pues una asociación entre género y acceso a la educación de las mujeres, en tanto poblaciones vulnerables y/o excluidas.

Este enfoque de género que se presenta en las conferencias y/o convenios evidencia el campo de tensiones y luchas permanentes a las que se enfrentan los distintos grupos para introducir esta perspectiva tan problemática y subversiva. Al revisar los convenios y las conferencias se observa que este enfoque no se presenta de manera sistemática y coherente. Por lo general, prima el denominado "feminismo de la igualdad", las conferencias no explicitan el enfoque de género. Es por ello que, en algunas situaciones, género equivale solo a mujeres y a acceso a la educación.

Asimismo, un aspecto que es importante relevar en la definición de las políticas educativas es la participación del movimiento feminista en el posicionamiento de la agenda de género en la educación. En el caso peruano, a diferencia de otros países de la región, el movimiento femi- 
nista no ha mostrado interés en la transformación del sistema educativo $y$, por lo tanto, no cuenta con una agenda para abordar este tema.

\section{Una mirada al currículo}

En el caso peruano, las estructuras curriculares, tanto de primaria como secundaria si bien cuentan con los denominados ejes transversales, su aplicación práctica no ha sido efectiva. Se observa que existe mayormente un uso "retórico" de tema de género. El tema se incorpora a título personal, dependiendo de la sensibilidad e interés de funcionarias y funcionarios por asumir género como tema. Es por ello que no hay espacios y mecanismos que garanticen un seguimiento y continuidad a las acciones emprendidas.

A diferencia de la estructura curricular de primaria, en el caso de secundaria, los contenidos transversales se han dejado abiertos bajo responsabilidad de los integrantes del centro educativo u organizaciones "representativas de la sociedad". Hubiera sido un gran aporte introducir de manera explícita algunos contenidos considerados de importancia, como el tema de género.

Asimismo, respecto a los contenidos de los materiales educativos, en el caso de primaria, la Directora Nacional planteó como un criterio a tomarse en cuenta que los textos se enmarcasen dentro de una perspectiva de género. La revisión del material producido, especialmente las guías de trabajo referidas al área de comunicación integral, mostró que se plantearon algunos cambios en relación al lenguaje, refiriéndose en algunos momentos a "niños y niñas". Parece que el hecho de explicitar el sexo a través de la diferencia de género en los sustantivos es asumida como una incorporación de la perspectiva de género.

Asimismo, esta inclusión no es sistemática. En algunos textos se hace referencia a "niños y/o niñas", planteando una diferenciación inclusiva con la que se reconoce la existencia de, por lo menos, dos géneros diferentes; en otros, se utiliza el genérico masculino, ya sea para referirse a quién habla (el autor, el libro): "Ahora invento una historia donde estos animalitos participen con el nombre que les he puesto. Pongo arriba un título y abajo mi nombre porque soy $e l$ autor" (Cuaderno de trabajo de comunicación integral para segundo grado de primaria), que representa a la vez a las personas a quienes va 
Caminos cruzados: Género en las políticas educativas en el Perú en los últimos diez años

dirigido el texto (los escolares). Es decir, existe una visión simplificada de género que es, a la vez, aplicada de manera poco sistemática.

Por otro lado, la presencia de la figura masculina continua siendo mayor. Por ejemplo, en las guías de segundo grado se han identificado 321 ilustraciones masculinas frente a 160 femeninas; y en las de tercer grado 341 ilustraciones masculinas y 190 femeninas. En el caso de las guías del sexto grado, se encontraron 329 ilustraciones masculinas frente a 269 femeninas. La prevalencia de hombres también fue observada por Jeanine Anderson en un estudio de revisión de textos escolares del año 1982 (Anderson y Herencia, 1982). En dicho trabajo la autora señalaba que este marcado sesgo a favor de figuras masculinas se constataba en la mayoría de los países donde se había realizado la revisión de textos.

Lo que llama la atención es que estas guías pese a que se inscriben en el marco de una nueva propuesta curricular - donde, al menos discursivamente, se menciona el tema de la equidad y el género - presenten los estereotipos tradicionales. Así por ejemplo, las ilustraciones que se observan son de niñas barriendo o lavando, mujeres en actitud protectora, cuidando a los niños, cargando a sus hijos para que les pongan las vacunas. Es decir, mujeres cumpliendo su rol doméstico y en la esfera de lo privado. Estas figuras se circunscriben a ambientes identificables con ésta: casa, hogar. Por el contrario, los hombres aparecen en el espacio público, trabajando, asumiendo roles profesionales como médicos o trabajadores de mando medio. En los pocos casos donde las mujeres aparecen como profesionales, éstas son representadas como maestras (haciéndose una alusión clara a la figura de la maestra como segunda madre). Por otro lado, los personajes que representan a la nación que aparecen en los textos son varones: héroes, guerreros y poetas. Asimismo, cuando se recrean la situación de las autoridades de una comunidad, éstas son representadas por hombres.

El tema de la eliminación de estereotipos en los textos escolares es mencionado por los funcionarios entrevistados como una experiencia puntual de aplicación de una perspectiva de género en las políticas públicas educativas. Esto no se evidencia en los materiales revisados. Podría señalarse que las alusiones de los entrevistados a la aplicación del enfoque de género podrían hacer referencia a experiencias puntuales, realizadas, sin coordinación alguna, en diferentes unidades del MED. 
Cabe señalar que, en el caso peruano, los materiales educativos se producen a través de distintas modalidades. A excepción de algunas direcciones - tales como la de primaria - que cuentan con una unidad encargada del desarrollo de los materiales, por lo general el Ministerio, a través de concursos de licitación, procede a la adquisición y/o producción de materiales educativos.

\section{Formación y capacitación docente}

Las propuestas de formación y capacitación docente que se aplican en el contexto de reforma durante este periodo son dos (1) El Proyecto de Modernización de la Formación Docente (PMFD), que pretendía incidir en la formación inicial de los docentes y (2) el Plan Nacional de Capacitación Docente (PLANCAD), desarrollado desde la Unidad de Capacitación Docente (UCAD), cuyo objetivo era capacitar a los y las docentes en ejercicio. Una revisión de ambas propuestas permitirá identificar la presencia (o ausencia) de un enfoque de género, las posibilidades que abrieron, las que cerraron o las que no se aprovecharon en el tema de la formación y capacitación docente.

Cabe destacar que si bien es cierto, éstos fueron los programas centrales del MED en este campo, otras Direcciones Nacionales y sus respectivas unidades ofrecieron otro tipo de capacitaciones, las cuales utilizaron "diversos enfoques, distintas estrategias y con objetivos específicos, muchas veces disímiles entre sî" (Cuenca, 2003, p. 41). Este dato es sumamente importante, ya que revela la poca capacidad que tiene el MED para centralizar tareas y una sobre oferta en el caso de la formación y capacitación docente. En una investigación sobre la oferta de capacitación docente se halló que "el 90\% de las unidades del MED ofrecieron y ofrecen capacitación a los docentes, ya sea en el marco de 'proyectos piloto o como acciones generalizadas'" (idem, 2002). Ambas propuestas estuvieron financiadas por organismos multilaterales, tales como el BM y el BIRF, así mismo, recibieron asesoría del proyecto proforma-GTZ de la Agencia de Cooperación Técnica Alemana (GTZ).

Los ejes temáticos en torno a los cuales giró el Proyecto Nacional de Formación Docente estuvieron relacionados con la re-configuración del marco teórico-pedagógico a partir del denominado nuevo enfoque pedagógico (NEP), la transformación de la gestión institucional (funciona- 
miento cooperativo entre ISP, vida cooperativa y democrática en la institución) y la re-configuración de la relación ISP-comunidad (diversificación, interacción con la comunidad).

La elaboración del nuevo currículo siguió un largo camino que se inició con un plan piloto, el cual se empezó aplicar hacia 1996 y contó con la participación de 15 institutos superiores pedagógicos en todo el país. En estos dos períodos el currículo se experimentó y validó para, luego, a partir del 2001, entrar en un proceso de generalización.

Las referencias al tema de género en este currículo son imprecisas y a veces contradictorias entre sí. Se le menciona, en primer lugar, como un tipo de formación que recibirán los docentes, el cual nunca se hace operativo en los carteles de contenido de áreas como sociedad, comunicación y ecosistema. Por otro lado, aparece, a la vez, como una perspectiva problemática en el cartel de contenidos del área de religión.

En cuanto al PLANCAD, este tenía como finalidad capacitar a los docentes en servicio en el nuevo enfoque pedagógico. Durante estos años se capacitaron a alrededor de 209.000 docentes (Cuenca, 2003) a través de instituciones de capacitación, denominadas entes ejecutores. ${ }^{7}$ Desde el Ministerio se señalaron los temas a trabajar, pero existió libertad para que cada institución tuviera su propia propuesta metodológica. Solo en el caso de secundaria se desarrolló una experiencia muy puntual de capacitación en género a un grupo de docentes que desarrollaban el Piloto de Secundaria.

Programas compensatorios: La Ley de Fomento de la Educación de las Niñas y Adolescentes Rurales (2001)

En el caso peruano, una de las experiencias que hay que relevar es la promulgación de la Ley de Fomento de la Educación de las Niñas y Adolescentes Rurales, que surge como respuesta a una iniciativa de la sociedad civil y de la cooperación internacional. En ese sentido, es importante destacar el papel que cumplió la Red - Florecer, Red para el Desarrollo de la Educación de las Niñas y Adolescentes Rurales (1998), en la elaboración, debate y posterior promulgación de esta ley.

La iniciativa de la Red fue impulsada desde la Agencia de Cooperación para el Desarrollo Internacional de los Estados Unidos (USAID). Posteriormente contó con la participación de diversos sectores de la so- 
ciedad civil. USAID, en el marco de sus prioridades orientadas al tema del desarrollo, formuló un proyecto global que incluiría a Perú, Marruecos, Egipto, Mali y Guatemala, denominado Educación de la Niña. Aunque este proyecto tenía la finalidad de impulsar la educación de las niñas por considerarlas un factor importante para el desarrollo, no se enmarcó en un enfoque de género.

Una característica de este proyecto fue el hecho de centrarse en buscar los medios y canales para propiciar cambios socio-políticos que influyeran en políticas dirigidas a apoyar la educación de las niñas. En el Perú, CARe-Perú es la institución que gana el concurso para dirigir el Proyecto Nuevos Horizontes para la educación de las niñas (PNHEN). Este proyecto comenzó a ejecutarse en abril de 1998 y concluyó en marzo del 2002 (ver más adelante).

La finalidad del PNHEN fue: "Mejorar las oportunidades y calidad de la educación primaria de las niñas de áreas rurales, a través de una estrategia nacional de concertación de líderes del sector público y de la sociedad civil" (CARE, 2002, p. 7). Las acciones de este proyecto estuvieron organizadas en tres aspectos: a) mejorar el conocimiento con relación a la educación de las niñas, b) inspirar políticas públicas, a través de la creación de una ley de fomento de la educación de la niña rural e impulsar la constitución de una red nacional de educación de la niña y una red departamental en Ayacucho (RENIRA); y c) la promulgación de una Ley de Fomento de la Educación de las Niñas y Adolescentes rurales.

Con la formulación de La Ley de Fomento, Ley n. 27558, se hace explícito el compromiso del Estado y la sociedad civil con la educación de las niñas y adolescentes rurales. El Estado y el gobierno peruano declaran al periodo 2002-2006 como el "Quinquenio de la educación Rural” (Diario Oficial El Peruano, 2001, art. 3º), señalándose que la prioridad de los recursos públicos debería ser orientada hacia ese sector.

La Ley promulgada consta de tres títulos: el primero que se denomina "De la equidad en la educación de las niñas, niños y adolescentes rurales"; el segundo, "De los objetivos y estrategias de la educación de las niñas y adolescentes rurales"; y uno tercero, "Profesores y participación familiar y comunal en la educación de las niñas y adolescentes en áreas rurales". El concepto de equidad utilizado en esta ley está asociado a las niñas y adolescentes rurales, en tanto población vulnerable: 
Caminos cruzados: Género en las políticas educativas en el Perú en los últimos diez años

El Estado promueve condiciones de equidad entre niños, niñas y adolescentes en áreas rurales para lo cual debe formular políticas educativas que respondan a las necesidades de ese sector y, específicamente, de las niñas y adolescentes rurales, en el marco de una formación integral y de calidad para todos. (Diario Oficial El Peruano, 2001, art. 1º)

En la Ley, la equidad de género esta entendida en términos de acceso, de calidad y de igualdad en el trato (idem, ibid., art. $6^{\circ}$, inciso b). Se trata, por ello, de formular medidas compensatorias. Estas medidas tratan de responder a (1) los problemas identificados con las niñas y adolescentes rurales: la matrícula, la extra-edad, la ausencia de servicios higiénicos diferenciados, el abuso sexual, (2) la promoción de la diversificación curricular de acuerdo con las distintas realidades socioculturales y (3) la búsqueda de la incursión de las niñas y adolescentes rurales en campos de los que estas se han visto excluidas, tales como los deportes, los certámenes, el liderazgo, entre otros.

Entre noviembre del 2001 - año en que se promulgó la Ley - y el 2002, se aplicaron acciones orientadas a implementarla. Éstas se inician con la constitución de un espacio de concertación para proponer y articular acciones interinstitucionales para el cumplimiento de los objetivos de la misma. Es así que el 23 de enero del 2003 se crea la Comisión Multisectorial de Fomento de la Educación de las Niñas y Adolescentes Rurales. Una de las actividades iniciales de esta Comisión fue el proceso de constitución de la comisión, organización y reglamento de funcionamiento, y la elaboración de los lineamientos para el plan de trabajo. Poco es lo que se viene avanzando en la Comisión Multisectorial, puesto que existe una fuerte resistencia al trabajo intersectorial.

Recebido em maio de 2006 e aprovado em junho de 2006.

Notas

1. Quiero expresar mi agradecimiento a José Luis Rosales y a Luciana Córdova, quienes participaron durante el desarrollo de la investigación en distintos momentos.

2. Estas reformas se el marco del Programa de Mejoramiento de la Calidad de la Educación Peruana (MECEP).

3. En la Conferencia de Beijing, en 1995, a través de una Plataforma de Acción elaborada por 189 países, se recomendó que los gobiernos garantizaran a las mujeres acceso a recur- 
sos y oportunidades educativas. En el Foro Mundial de la Educación en Dakar, entre el 26 y el 28 de abril del 2000, se evaluó que, si bien se habían logrado algunos objetivos, todavía faltaba alcanzar otros. En esta reunión se coincidió en mirar la educación como un derecho y se puso mayor énfasis en la eliminación de las disparidades de género, tanto en la educación primaria como en la secundaria (Torres, 2000; CEPAL, 2004).

4. La elaboración de este documento estuvo a cargo de la Oficina de Planificación Estratégica del MED, la cual intentó coordinar con otras oficinas de la Sede Central del Ministerio de Educación. Con este diagnóstico se hizo evidente que el Perú no realizó acciones para promover la equidad de género en sus políticas educativas (UNESCO y TAREA, 2001, p. 55).

5. Con tal fin, se señala que el Estado combatirá "la discriminación por razones de inequidad entre hombres y mujeres, origen étnico, raza, credo o discapacidad" (Acuerdo Nacional, 2002: Décima Política de Estado).

6. Cabe destacar que recién el año 2005, el Perú publicó Plan Nacional de Educación para Todos (Ministerio de Educación, Foro Nacional de Educación para Todos, 2005). Aunque este último documento no ha sido incorporado en el análisis es interesante anotar que se indica que la erradicación de las desigualdades de género es un enfoque transversal a todos los objetivos estratégicos. Y por equidad de genero se entiende como un "principio de justicia que trata de eliminar todas las barreras que impiden la igualdad de oportunidades económicas, políticas, de acceso a la educación, a los recursos y a los servicios básicos entre hombres y mujeres. Equidad de género connota la igualdad de derechos" (Plan Nacional de Educación para Todos, 2005, p. 80).

7. Los denominados "entes ejecutores" fueron instituciones de la sociedad civil e institutos superiores pedagógicos que participaron en esta tarea.

\section{Referencias bibliográficas}

ACUERDO NACIONAL. Décima politica de Estado. 2002. Disponible en: <www.acuerdonacional.gob.pe>

ANDERSON, J.; HERENCIA, C. La imagen de la mujer y del varón en los libros escolares peruanos. Lima: UNESCO, 1982. (mimeo)

BARRIG, M. La persistencia de la memoria: feminismo y Estado en el Perú de los noventa. Lima: PUCP, 2000.

BLONDET, C. El encanto del dictador: mujeres y política en la década de Fujimori. Lima: IEP, 2002.

BUTLER, J. El género en disputa: el feminismo y la subversión de la identidad. México, DF: Paidós, 2001.

CARE. Nuevos horizontes para la educación de las niñas. Lima: CARE, 2002.

CLADEM. Diagnóstico sobre la situación de los derechos sexuales y los derechos reproductivos: 1995-2002. Lima: CLADEM, 2006. 
Caminos cruzados: Género en las políticas educativas en el Perú en los últimos diez años

COMISION ECONOMICA PARA AMERICA LATINA Y EL CARIBE (CEPAL). Novena conferencia regional sobre la mujer de América latina y el Caribe. 2004. Folleto informativo. Disponible en: <www.cepal.cl>. Aceso en: 2004.

CONSEJO NACIONAL DE EDUCACIÓN. Pacto social de compromisos recíprocos por la educación. El Comercio, Lima, suplemento 2004.

CUENCA, R. La oferta de capacitación docente en el Ministerio de Educación. Lima: GTZ, 2002.

CUENCA, R. El compromiso de la sociedad civil con la educación: sistematización del Plan Nacional de Capacitación Docente (PLANCAD). Lima: Ministerio de Educación; GTZ; KFW; 2003.

DURAND, F. Riqueza económica y pobreza politica: reflexiones sobre las elites del poder en un país inestable. Lima: PUCP, 2003.

FORO MUNDIAL SOBRE LA EDUCACIÓN. Educación para todos: cumplir nuestros objetivos comunes; texto aprobado por el Foro Mundial sobre la Educación, Dakar, Senegal, 26-28 de abril del 2000. Disponible en: <www.unesco.org>.

FRANCKE, P. Politicas sociales: balance y alternativas. Lima, 2003. (mimeo).

FRASER, N. Institia interrupta: reflexiones críticas desde la posición "postclasista”. Bogotá: Universidad de los Andes; Siglo del Hombre, 1997.

IRIGARAY, L. Ser dos. México, DF; Argentina: Paidos, 1998. p. 88.

LAZARTE, C.; LANZA, M. Equidad de género en las políticas educativas bolivianas: experiencias y desafíos. In: CorTINA, R.; STROMQUist, N. Promoviendo la educación de mujeres y niñas en América Latina. México, DF: Pax, 2001.

LÓPEZ, N. Equidad educativa y desigualdad social: desafíos a la educación en el nuevo escenario latinoamericano. Buenos Aires: Instituto Internacional de Planeamiento de la Educación (IIPE); UNESCO, 2005.

MONTERO, C.; TOVAR, T. Agenda abierta para la educación de las niñas rurales. Lima: CARE; IEP; Foro Educativo, 1996. 
MUÑOZ, F.; ROSALES, J.L. Una mirada a las agencias de cooperación internacional: las potencialidades y límites para atender la educación en áreas rurales (1996-2003). In: LA NUEVA ruralidad: desafíos y propuestas; ponencias presentadas en el Seminario Internacional. Lima: Ayuda en Acción. 2004.

PERU. Constitución Política del Perú de 1993. Disponible en: <www.congreso.gob.pe>. Aceso en: 2004.

PERU. Grupo de Análisis para el Desarrollo y Symmetry Systems. Mejoramiento de los Procesos de Desarrollo Curricular y Material Educativo. Segundo Informe: Avances de la Consultoría. Servicio de Consultoría no 010-2000-ED/MECEP. Lima: Ministerio de Educación, 2000.

PERU. Ley n. 23384, publicada el 20 de mayo de 1982. Ley General de Educación. Diário Oficial El Peruano, Lima, 1982.

PERU. Decreto Supremo n. 001-2000-PROMUDEH, publicado el 1 de febrero del 2000. Plan Nacional de Igualdad de Oportunidades para Hombres y Mujeres. Diario Oficial El Peruano, Lima, 2000.

PERU. Ley n. 27558, publicada el 22 de noviembre del 2001. Ley de fomento de la educación de las niñas y adolescentes rurales. Diário Oficial El Peruano, Lima, 2001.

PERU. Resolución Ministerial n. 168-2002-ED, publicada el 14 de marzo de 2002. Aprueban disposiciones complementarias de las Normas para la Gestión y Desarrollo de las Actividades en los Centros y Programas Educativos. Diario Oficial El Peruano, Lima, 2002.

PERU. Resolución ministerial n. 0310-2003-ED, publicada el 3 de marzo del 2003. Orientaciones para el desarrollo de la actividad educativa para el año 2003. Diario Oficial El Peruano, 2003a.

PERU. Decreto Supremo n. 001-2003 -ED, publicado el 24 de enero del 2003. Se crea la Comisión Multisectorial de Fomento de la Educación de las Niñas y Adolescentes Rurales. Diario Oficial El Peruano, 2003b.

PERU. Resolución Ministerial n. 0440-2003-ED, publicada el 3 de abril del 2003. Designan entidad de asesoría técnica ad honorem de 
Caminos cruzados: Género en las políticas educativas en el Perú en los últimos diez años

la Comisión Multisectorial de Fomento de la Educación de las Niñas y Adolescentes Rurales. Diario Oficial El Peruano, Lima, 2003c.

PERU. Ley n. 28044, publicada el 29 de julio del 2003. Ley General de Educación. Diário Oficial El Peruano, Lima, 2003d.

PERU. Ministerio de Educación. Informe General de la Reforma de la Educación Peruana. Lima: MED, 1970.

PERU. Ministerio de Educación. Diagnóstico General de la Educación; Perú: calidad, eficiencia, equidad: los desafíos de la educación primaria. Lima: MED, Banco Mundial, PNUd, GTZ; UnESCO/OREALC, 1993.

PERU. Ministerio de Educación. Plan de Mediano y Largo Plazo (1995-2010): resumen ejecutivo; documento de trabajo. Lima: MED, 1996.

PERU. Ministerio de Educación. Nueva estructura del sistema educativo peruano: fundamento del sistema educativo peruano; fundamentos de la propuesta. Lima: MED, 1997a.

PERU. Ministerio de Educación. Currículo de formación docente: especialidad de educación primaria. Lima: MED, 1997b. Disponible en: $<$ www.minedu.gob.pe>. (mimeo)

PERU. Ministerio de Educación. Plan de mediano y largo plazo de la educación peruana 1998-2021; borrador de trabajo. Lima: MED, 1998. v.4 (mimeo).

PERU. Ministerio de Educación. Programa Curricular del Tercer ciclo de educación Primaria (quinto y sexto grado): estructura curricular básica de educación primaria de menores. Lima: MED, 1999.

PERU. Ministerio de Educación. El desarrollo de la educación. Lima: MED, 2001a.

PERU. Ministerio de Educación. Las voces del país. Resultados de la Consulta Nacional de Educación. Las puertas siguen abiertas. Lima, Suplemento contratado en diario El Comercio, 2001b.

PERU. Ministerio de Educación. Propuesta curricular experimental. Lima: MED, 2001c. 
PERU. Ministerio de Educación. Educación para la democracia: lineamientos de política educativa 2001-2006. Lima, Suplemento contratado en diario El Comercio, 13 ene. 2002.

PERU. Ministerio de Educación. Plan Nacional de Educación para Todos: documento base. Lima: MED, 2003 a.

PERU. Ministerio de Educación. Proyecto de educación en áreas rurales: plan de implementación. Lima: MED, 2003b. (mimeo).

PERU. Ministerio de Educación. Proyecto de educación en áreas rurales: estudio de prefactibilidad. Lima: MED, 2003c. (mimeo).

PERU. Ministerio de Educación. Plan de trabajo de la Comisión Multisectorial de Fomento de la Educación de las Niñas y Adolescentes Rurales: documento preliminar. Lima: MED, 2005. (mimeo).

PERU. Ministerio de Educación. Plan EPT: versión preliminar. Lima: MED, 2005. (mimeo).

PERU. Ministerio de Educación. Plan Nacional de Educación para Todos: hacia una educación de calidad con equidad. Foro Nacional de Educación para Todos, 2005.

PORTOCARRERO, F.; SANBORN, C.; CUEVA HANNY Y MILLÁN, A. Más allá del individualismo: el tercer sector en el Perú. Lima: Universidad del Pacífico, 2002.

PORTOCARRERO, G. Las relaciones Estado-sociedad en el Perú: un examen bibliográfico. Lima: PUCP, 2002.

RODRIGUEZ, J.; ZAMBRANO, G. Cobertura y aprendizajes en el Sistema Educativo Peruano. Lima, 2003. (mimeo).

RUIZ-BRAVO, P. De la protesta a la propuesta: itinerario de la investigación sobre relaciones de género. In: DEGREGORI, C.I (Ed.). Tiempos de ira y amor: nuevos actores para viejos problemas. Lima: DESCO, 1990.

SALAZAR HERRERA, C. Politica de mujeres peruanas durante el siglo $X X$ : tentando una cronología. Lima: Manuela Ramos, 2001. v.1

SCOTT, J. El género: una categoría útil para el análisis histórico. In: Lamas, M. (Comp.). El género: la construcción cultural de la diferencia sexual. México, DF: UNAM, 1997. 
Caminos cruzados: Género en las políticas educativas en el Perú en los últimos diez años

TORRES, R.M. ¿Qué pasó en el Foro Mundial de la Educación? Dakar, Senegal, 26-28 de abril de 2000. Disponible en: <www.fronesis.org>. (2004) [2000].

TOVAR, T. Las mujeres están queriendo igualarse: género en la escuela. Lima: TAREA, 1997.

UNESCO. Una mirada a la educación en el Perú: balance de 20 años del proyecto principal de la UNESCO para América Latina y El Caribe, 1979-1999. Lima: UNESCO; TAREA, 2001.

\section{Textos escolares analizados}

TRIN TRIN PALABRÍN; Cuaderno de trabajo; Comunicación integral. Lima: Ministerio de Educación, 2002.

MINKA. Cuaderno de trabajo: Comunicación integral; Segundo grado de educación primaria. Lima: MED; Brasa, 2000.

MINKA. Guía 2: Segundo grado de educación primaria; para el uso del cuaderno de trabajo del área de comunicación integral. Lima: MED; Brasa, sd.

MINKA. Cuaderno de trabajo: tercer, cuarto y quinto grado de educación primaria; Comunicación integral. Lima: MED; Brasa, 2000.

MINKA. Guía 5: Quinto grado de educación primaria; para el uso del cuaderno de trabajo del área de comunicación integral. Lima: MED; Brasa, sd.

MINKA. Cuaderno de trabajo: Sexto grado de educación primaria; Comunicación Integral. Lima: MED; Brasa, 2000.

MINKA. Guía 6: Sexto grado de educación primaria; para el uso del cuaderno de trabajo del área de comunicación integral. Lima: MED; Brasa, sd. 